\title{
The risk-return tradeoff: A COGARCH analysis of Merton's hypothesis
}

\author{
Gernot Müller ${ }^{\mathrm{a}, *}$, Robert B. Durand ${ }^{\mathrm{b}}$, Ross A. Maller ${ }^{\mathrm{c}, \mathrm{d}}$ \\ a Zentrum Mathematik, Technische Universität München, Boltzmannstraße 3, 85748 Garching, Germany \\ b The University of Western Australia, 35 Stirling Highway, Crawley WA 6009, Australia \\ c School of Finance \&' Applied Statistics, The Australian National University, ACT 0200, Australia \\ d Center for Mathematics \& its Applications, The Australian National University, ACT 0200, Australia
}

\section{A R T I C L E I N F O}

\section{Article history:}

Received 24 May 2009

Received in revised form 19 October 2010

Accepted 9 November 2010

Available online 16 November 2010

\section{JEL classification:}

C22

G12

\section{Keywords:}

Continuous-time GARCH modelling

Market risk

Pseudo-maximum likelihood

Risk free rate

Risk premium

Stochastic volatility

\begin{abstract}
A B S T R A C T
We analysed daily returns of the CRSP value weighted and equally weighted indices over 19532007 in order to test for Merton's theorised relationship between risk and return. Like some previous studies we used a GARCH stochastic volatility approach, employing not only traditional discrete time GARCH models but also using a COGARCH - a newly developed continuous-time GARCH model which allows for a rigorous analysis of unequally spaced data. When a risk-return relationship symmetric to positive or negative returns is postulated, a significant risk premium of the order of 7-8\% p.a., consistent with previously published estimates, is obtained. When the model includes an asymmetry effect, the estimated risk premium, still around 7\% p.a., becomes insignificant. These results are robust to the use of a value weighted or equally weighted index. The COGARCH model properly allows for unequally spaced time series data. As a sidelight, the model estimates that, during the period from 1953 to 2007, the weekend is equivalent, in volatility terms, to about $0.3-0.5$ regular trading days.
\end{abstract}

(c) 2010 Elsevier B.V. All rights reserved.

\section{Introduction}

Finance theory rightly maintains a deep and abiding preoccupation with the relationship between risk and return. So important and fundamental is this that any means of shedding light on it should be vigorously pursued. In the present paper we use a newly developed continuous time GARCH-like model (the "COGARCH"), as well as more traditional discrete time GARCH models, on a large data set, to advance our understanding.

At the time of writing, when fallout from the "Global Financial Crisis" is among the chief priorities of governments around the world, we are compelled to reconsider the connection between risk and return. ${ }^{1}$ Merton (1980) argued that there should be a positive relationship between the expected market risk premium - the return of the market less the risk-free rate of return - and the expected volatility of the market's returns. Put crudely, his thesis is that investors require a bribe in the form of higher potential returns to take on extra risk. Merton's analysis is an important foundational underpinning of theoretical finance, and, in the commercial world, a positive return to risk relationship underlies almost all of the advice investors receive from their advisors. But does such a relationship really exist?

The major stumbling block in testing Merton's model has been estimating expected risk. Researchers face a "chicken and egg" problem. Merton's proposition states that returns are a function of volatility, yet volatility must, in some way, be estimated using

\footnotetext{
* Corresponding author.

E-mail addresses: mueller@ma.tum.de (G. Müller), Robert.Durand@uwa.edu.au (R.B. Durand), ross.maller@anu.edu.au (R.A. Maller).

1 From the end of June 2007, when two of Bear Sterns' hedge funds suffered heavy losses due to their exposure to the sub-prime market, to the end of 2008, the S\&P 500 fell by approximately $40 \%$.
} 
returns. ${ }^{2}$ Stochastic volatility models are a natural choice with which to model the fluctuations observed in returns volatility, and among this class, conditional volatility models have proven popular. Yet a number of studies utilizing one of the many variant conditional volatility models have found, at best, only weak empirical evidence supporting a positive relationship between the market risk premium and volatility (see, e.g., French et al., 19873; Baillie and DeGennaro, 1990), or mixed evidence (see, for example, Campbell and Hentschel, 19924; Glosten et al., 1993); or even a negative relationship (see, e.g., Nelson, $1991^{5}$; Guo and Whitelaw, $2006^{6}$ ). Perhaps, as argued by Jorion and Goetzmann (1999), the signal to noise ratio is simply too weak to be detected in studies like these. ${ }^{7}$ Ang and Liu (2007), however, demonstrate that such mixed findings are to be expected; for example, the risk-return relation can be negative when dividend yields behave in the way modelled by Cox et al. (1985). Scruggs (1998) has argued that the difficulty in confirming Merton's predicted positive relationship between return and risk is a function of an omitted state variable. Investors' ex ante expectations about returns may be conditioned on ex post information sets capturing investment opportunities and consumption preferences. Lewellen and Nagel (2006) remind us, however, that tests are "strictly valid only if the econometrician knows the full set of state variables available to investors" (p. 296). ${ }^{8}$ Eschewing stochastic volatility models in their analysis confirming a positive risk-return trade-off, Ghysels et al. (2005) utilize the MIDAS methodology which uses a rolling window method combining both daily and monthly data, in which the window length is implicitly chosen in an optimal manner. It performs better than GARCH in their analyses, which they attribute to the fact that the GARCH model is only fitted to (equally spaced) monthly data. Ghysels et al.'s criticism of the use of GARCH for the analysis of irregularly spaced data is pertinent for our paper as the COGARCH approach we advocate explicitly allows for this.

After something of a hiatus in the use of conditional volatility models in this context, Lundblad (2007) revisited the problem and, in addition to finding a positive relationship between return and risk, provided an indication of why previous studies have had varied results. Lundblad examines Merton's propositions using estimates of variance calculated from a variety of models - GARCH $(1,1), \operatorname{EGARCH}(1,1), \mathrm{QGARCH}(1,1)$ and TARCH$(1,1)$ - and finds evidence in support of it. ${ }^{9}$ Even in this study, however, the evidence in favour of Merton is not strong. Using Monte Carlo simulations, Lundblad demonstrates how previous studies may have been hampered by small sample problems. To examine Merton's proposition with tests of adequate power, a large sample is essential. Lundblad addresses this issue by using monthly data spanning the period from the 1830s to the early 2000s. While this represents one way of increasing the power of tests, it is not necessarily the only course of action. The analysis assumes stationarity in the data and this might be considered a heroic assumption given the length of the time period over which the relationship is analysed. Lundblad's approach does have the advantage of using monthly data, as appears to be standard in tests of asset pricing models.

In the present paper we analyse an extensive dataset to estimate conditional volatility, and relate it to excess market return. Our principle analysis focuses on the period 1953 to 2007, which satisfies the requirements of Merton (1980), who argued that a long time span is needed to capture expected return variation, and our use of daily data is consistent with Lundblad's recommendation for a large sample (we have 13,844 daily returns in each of two series, see Section 3 ). ${ }^{10} 1953$ was chosen as a start date for our main analysis as it is the first complete year of data without trading on Saturdays. This means that we have a second set of daily data, from 1927 to 1951, when trading occurred on Saturdays, to test the robustness of the inferences we make on the basis of the analysis of the later period. ${ }^{11}$

The use of daily data enables us to consider the fine structure of volatility (lost when monthly data is used), but it introduces further problems in the form of daily seasonalities (such as Friday effects) and the effect of discontinuities in the data (such as weekends and holidays when there is no trading), which should be considered in a thoroughgoing analysis. The COGARCH model of Klüppelberg et al. (2004) lends itself naturally to our problem. COGARCH is a continuous-time version of a GARCH model, which preserves the important GARCH feature of feedback between returns and volatility, but is well adapted to handling unequally spaced time series data. For example, $\mathrm{COGARCH}$ has been used to model the daily volatility of ten years of data from the Australian stock market (Maller et al., 2008), taking into account weekend and holiday effects, and Müller et al. (2009) use it to model the volatility of the S\&P 500 using readings taken at 5 min intervals from 1998 to 2007.

\footnotetext{
${ }^{2}$ Other research has avoided the problem of the joint endogeneity of returns and risk by using an exogenous proxy such as the Chicago Board Options Exchange Volatility Index (the VIX) (see, for example, Ang et al., 2006; Durand et al., 2011). Durand et al. (2011) argue that a negative correlation between market returns and unexpected changes in volatility is associated with a repricing of risk. Such an interpretation is natural if prices in securities markets are thought of as the present value of future cash flows where the present value is calculated using a risk-adjusted discount rate. Any increase in expected risk will increase the discount rate and, hence, reduce the price.

${ }^{3}$ French et al., however, find a negative relationship of unexpected changes in volatility to returns which is supported by Durand et al. (2011).

${ }^{4}$ Campbell and Hentschel argue that volatility only matters when it is relatively high.

${ }^{5}$ Nelson finds a negative relationship in some, but not all, instances.

${ }^{6}$ Guo and Whitelaw, however, focus on Merton (1973) and also consider the investment opportunity set. The relationship studied in the present paper, and in Lundblad (2007), is based on that presented in Merton (1980) which represents a simplification of those in his 1973 paper.

${ }^{7}$ We are reminded en passant of the difficulty of finding any relationship between return and risk in a recent paper: Pástor et al. (2008), in their study of the relationship of risk and return to the cost of capital, do not report their analyses of the relationship of the market risk premium to volatility, a necessarily prelude to their analysis, in order to "save space" as "the results are disappointing" (p. 2879).

${ }^{8}$ Chen (1991) presents a seminal work in which state variables are linked to past, present and future economic states and stock returns. An additional problem faced in the analysis presented in this paper, which uses daily data, is that the "usual suspects" such as industrial production and inflation for state variables are not available at the frequency required.

${ }_{9}^{9}$ Lundblad's results are robust to the model used to estimate conditional volatility. He is "agnostic" as to the best functional form of the model.

${ }^{10}$ Andersen and Bollerslev (1998) show that the use of even higher frequency intraday data improves volatility estimation, but Lundblad's (2007) simulation investigation suggests that little extra would be gained in our context by taking even larger samples. There would however be no computational difficulty in extending our analyses to intra-day data. As a compromise we decided to use daily data over a reasonably long time span.

${ }^{11}$ We use only years where we have data for all of the year. Therefore, we do not use data from 1952 as Saturday trading ended during that year.
} 
To maintain comparability with Lundblad's and other published results, we also analyse weekly and monthly data drawn from the same sources, and, besides the basic COGARCH model, we consider also modifications of the main COGARCH specification as well as some other well-known discrete time GARCH models. In particular, the asymmetric effect of volatility (whereby a positive return may be related to volatility in a different way to a negative return) at the firm and market levels has been documented (see, for example, Bekaert and $\mathrm{Wu}, 2000^{12}$ ) and, following this lead, we extend the COGARCH model to consider such asymmetric effects. In our analysis, presented in Section 4 of this paper, the asymmetric COGARCH model proves to be the preferred model using either the Akaike Information Criterion (AIC) or the Bayesian Information Criterion (BIC), but the asymmetric COGARCH model is not supportive of Merton's hypothesis. Merton's proposition is, however, supported by the symmetric version (positive and negative returns have the same relationship to volatility) of the $\mathrm{COGARCH}$ analysis; that is, we find significant positive covariance between the market riskpremia of both the CRSP value-weighted and equal-weighted excess market returns, and their volatilities, in the symmetric COGARCH model. $^{13}$

The COGARCH models are set out in Section 2, our data set is described in detail in Section 3, results are in Section 4, and we conclude in Section 5.

\section{Methodology}

Merton (1980) proposed a linear relation between the conditional mean of the return of the wealth portfolio and its conditional variance. To formulate the models we consider, suppose we are given $N$ observations on an asset price process over a time interval [0,T], observed at not necessarily equally spaced times $0=t_{0}<t_{1}<\cdots<t_{N}=T$. Let $R_{i}$ denote the return, and $r_{f, i}$ the risk-free rate, at time $t_{i}$, and let $\sigma_{i}^{2}$ be the variance of $R_{i}$, conditional on past information $\mathcal{F}_{t_{i-1}}$; thus, $\sigma_{i}^{2}=\operatorname{Var}\left(R_{i} \mid \mathcal{F}_{t_{i-1}}\right)$.

Our basic model for excess returns is then

$$
Y_{i}=R_{i}-r_{f, i}=\lambda_{1}+\lambda_{2} \sigma_{i}^{2}+\sigma_{i} \varepsilon_{i}, \quad i=1, \ldots, N
$$

where $\lambda_{1}$ and $\lambda_{2}$ are parameters to be estimated, the random variables $\varepsilon_{i}$ are assumed to be independent with mean 0 and variance 1 , and we will identify $\sigma_{i} \varepsilon_{i}$ with the increments of a (possibly asymmetric) COGARCH process; see Section 2.1 and Appendix A for

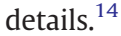

Our Eq. (1), like Lundblad's Eq. (3), allows a general relationship between return and risk, based on a linear regression between excess returns (we use the returns of the market proxy less the risk free rate), and the variance. These equations are more general than Merton's Model 1 (see Merton (1980), pp. 329-330), in which he proposes that the market risk premium is a function of the variance of returns, and that the coefficient corresponding to the variance may be interpreted as “...the reciprocal of the weighted sum of the reciprocal of each investor's relative risk aversion and the weights are related to the distribution of wealth among investors" (Merton, 1980, p. 329). Eq. (1) includes an intercept coefficient $\lambda_{1}$, and, as a result of the divergence from the strict formulation of Merton's proposition, $\lambda_{2}$ may not be given such a strict economic interpretation as that proposed by Merton. ${ }^{15}$ When we estimate Eq. (1), we will denote it as COGARCH (U) ("U" for "unitary" time scale). A particular advantage of COGARCH, however, is that we can switch over to a virtual time scale to account for weekday effects (for daily data), weekly effects (for weekly data) or monthly effects (for monthly data); we discuss this further in Section 2.2 below. We will denote the resulting model by COGARCH (V), "V" denoting "virtual time scale" (when taking weekday and month effects into account). We extend COGARCH (U) and COGARCH (V) to account for the possibility of the presence of an asymmetric relationship of return and risk and denote these extensions to the COGARCH models as asyCOGARCH (U) and asyCOGARCH (V) (details of how the models are extended to account for asymmetry may be found in Appendix A).

We compare nested models using AIC and BIC. Instead of judging only from the likelihood, these criteria punish more complex models by a penalty term. The model with the lower AIC and/or BIC is to be preferred. We emphasise that only nested models can be compared using these criteria. For non-nested models a comparison of the AIC and BIC values can be misleading. Thus, the models COGARCH (U)-COGARCH (V), COGARCH (V)-asyCOGARCH (V), COGARCH (U)-asyCOGARCH (U) and asyCOGARCH (U)-asyCOGARCH (V) are nested, and hence, comparable by means of AIC and BIC. The asyCOGARCH (U)-asyCOGARCH (V) comparison enables us to decide whether we achieve a significant improvement by working on a virtual time scale. We cannot, however, compare asyCOGARCH (U) with COGARCH (V) using AIC or BIC.

Our goal is not only to estimate the impact of the volatility on the mean of the excess return, but at the same time to investigate the fine structure of the volatility. Whereas Lundblad (2007) used GARCH, EGARCH, QGARCH, TARCH, specifications of the conditional variance component, we assume that the process follows a COGARCH model. The most important feature of the COGARCH model is that it is formulated in continuous time. This provides great flexibility and accuracy in modelling. In particular, COGARCH is well suited to the analysis of irregularly spaced time series data with a GARCH-like volatility structure. As we will see later we are also able to take into account exogenous variables which may have an impact on volatility.

\footnotetext{
12 Table 1 on page 3 of this paper provides a useful summary of findings relating asymmetric volatility and returns.

13 Our results compare well with those published in a variety of sources. We take the figures for comparison from Table 1 , p. 504 , of Welch, 2000.

14 We also considered a variant of Eq. (1) which departed from Merton and Lundblad in using lagged conditional volatility as the dependent variable. Consideration of this model did not lead to materially different inferences from than those we make on the basis of our estimates of Eq. (1) so we do not report further on those findings in this paper.

${ }^{15}$ By way of interest, Merton's Model 2 replaces variance with standard deviation and the covariate of the standard deviation may be interpreted as the market price of risk. We do not consider this model. Merton's Model 3 simply proposes that the excess return is constant.
} 
The COGARCH model is relatively new to finance and consequently we provide some background information on it in Section 2.1 and describe the pseudo-maximum-likelihood (PML) methodology used in its estimation in Appendix A.

\subsection{The COGARCH methodology}

We recall the definition of the COGARCH $(1,1)$ process (hereafter referred to simply as a COGARCH process) ${ }^{16}$ introduced in Klüppelberg et al. (2004). In the COGARCH, both the return and volatility depend only on a single background driving Lévy process $L=$ $(L(t))_{t \geq 0}$, satisfying $\mathbb{E} L(1)=0$ and $\mathbb{E} L^{2}(1)=1$. See Applebaum (2004), Bertoin (1996), and Sato (1999) for detailed results concerning Lévy processes.

The COGARCH variance process $\rho^{2}=\left(\rho^{2}(t)\right)_{t \geq 0}$ is defined as the almost surely unique solution of the stochastic differential equation

$$
\mathrm{d} \rho^{2}(t)=\left(\beta-\eta \rho^{2}(t-)\right) \mathrm{d} t+\varphi \rho^{2}(t-) \mathrm{d}[L, L](t), t>0,
$$

where $[L, L]$ is the bracket process (quadratic variation) of $L$ (Protter, 2005, p. 66). Eq. (2) is closely related to a discrete time GARCH recursion; see Eq. (2.8) of Maller et al. (2008). Parameters $(\beta, \eta, \varphi)$ satisfy $\beta>0, \eta>0, \varphi \geq 0$, and the initial volatility, $\rho(0)$, is a finite variance random variable independent of $L$. Eq. (2) can be solved to reveal $\rho^{2}(t)$ as a mean reverting process, in fact, a kind of generalised Ornstein-Uhlenbeck process (see Eq. (2.13) of Maller et al., 2008).

Eq. (2) treats returns symmetrically in the sense that current volatility is related to past volatility via the single parameter $\varphi \geq 0$, and the quadratic variation differential $\mathrm{d}[L, L](t)$, also non-negative, which plays the role of $\varepsilon_{i}^{2}$, where $\varepsilon_{i}$ is as in Eq. (1). To allow for an asymmetric feedback effect of return on volatility, we split parameter $\varphi$ into two parameters, $\varphi^{+}$, which factors in a contribution from a positive return, and $\varphi^{-}$, which factors in a contribution from a negative return. The precise method of doing this is detailed in Appendix A.

Our model for the log asset price process (discounted for the risk-free rate) is then the integrated COGARCH process $G=(G(t))_{t \geq 0}$ defined in terms of $L$ and $\rho$ as

$$
G(t)=\int_{0}^{t} \rho(s-) d L(s), \quad t \geq 0 .
$$

As shown in Klüppelberg et al. (2004), Corollary 3.1, both $(\rho(t))_{t \geq 0}$ and the bivariate process $(\rho(t), G(t))_{t \geq 0}$ are Markovian. Moreover, under a certain integrability condition, and for a choice of $\rho^{2}(0)$ satisfying $\mathbb{E} \rho^{2}(0)=\beta /(\eta-\varphi)$, with $\eta>\varphi$, the process $\rho^{2}(t)$ is strictly stationary (Klüppelberg et al., 2004, Thm. 3.2), and then $(G(t))_{t \geq 0}$ has stationary increments.

While COGARCH allows us to model complex data, it is a relatively parsimonious model requiring estimation of only three (or four) parameters: $\beta, \eta$ and $\varphi$ (or $\varphi^{ \pm}$). To help with an intuitive understanding of these parameters, we recall that, when the driving Lévy process $L$ is compound Poisson, the COGARCH volatility is exponentially decreasing between upward jumps occurring at exponentially distributed times (Fasen et al., 2005). Parameter $\eta$ is the rate at which the volatility decreases exponentially between jumps, while $\varphi$ relates to the magnitude of the jumps between these decreasing periods. $\beta$ is related to but does not directly measure the overall mean level of volatility; similar to the discrete time GARCH, the long-run average of volatility is computed as $\beta /(\eta-\varphi)$.

It can be shown that both the tail of the distribution of the stationary volatility and the tail of the distribution of $G(t)$ are Pareto-like under weak assumptions (cf. Klüppelberg et al., 2006). Thus the COGARCH distribution tails are "heavy", consistent with recent extensive empirical evidence of Platen and Sidorowicz (2007). For more details on the theoretical properties of $G$ and $\rho^{2}$, we refer to Klüppelberg et al. (2004) and Klüppelberg et al. (2006). Fasen et al. (2005) show that the COGARCH model, in general, exhibits regularly varying (heavy) tails, volatility jumps upwards, and clusters on high levels.

The data fitting procedure is operationalised as follows. For the pure COGARCH process, we wish to estimate just the parameters $(\beta, \eta, \varphi)$. Suppose we are given observations $G\left(t_{i}\right)$, at times $0=t_{0}<t_{1}<\cdots<t_{N}=T$, on the log price process, as modelled by the integrated COGARCH defined and parameterised in Eqs. (2) and (3), assumed to be in its stationary regime. The $\left\{t_{i}\right\}$ are assumed fixed (non-random) time points. Set $\Delta t_{i}:=t_{i}-t_{i-1}$ and let $Y_{i}=G\left(t_{i}\right)-G\left(t_{i-1}\right), i=1, \ldots, N$, denote the returns. From Eq. (3) we can write

$$
Y_{i}=\int_{t_{i-1}}^{t_{i}} \rho(s-) \mathrm{d} L(s)
$$

The Lévy Process $L$ has stationary independent increments which are analogous to the i.i.d. innovations in a regression or discrete time GARCH process. From Eq. (3), the infinitesimal increment $\mathrm{d} G(t)$ equates to $\rho(t-) \mathrm{d} L(t)$, while by Eq. (4), the discrete increment $Y_{i}$ approximates $\rho\left(t_{i-1}\right) \Delta L_{i}$, where $\Delta L_{i}=L\left(t_{i}\right)-L\left(t_{i-1}\right)$. A crucial feature of the COGARCH is, as in the discrete time GARCH, the presence of feedback between the returns, $Y_{i} \approx \rho\left(t_{i-1}\right) \Delta L_{i}$, and the volatility, as a function of $\left(\Delta L_{i}\right)^{2}$ (by way of the quadratic variation term in Eq. (2)).

\footnotetext{
$\overline{{ }^{16} \operatorname{COGARCH}}(1,1)$ has been extended to COGARCH $(\mathrm{p}, \mathrm{q})$ in Brockwell et al. (2006), and a multivariate version is formulated in Stelzer (2009). For our purposes,
} the $(1,1)$ model suffices. 
The $Y_{i}$ have conditional expectation 0 and conditional variance

$$
\sigma_{i}^{2}:=\mathbb{E}\left(Y_{i}^{2} \mid \mathcal{F}_{t_{i-1}}\right)=\left(\rho^{2}\left(t_{i-1}\right)-\frac{\beta}{\eta-\varphi}\right)\left(\frac{e^{(\eta-\varphi) \Delta t_{i}}-1}{\eta-\varphi}\right)+\frac{\beta \Delta t_{i}}{\eta-\varphi} ;
$$

see Eq. (2.8) of Maller et al. (2008). The residual term in Eq. (1) is constructed as $\sigma_{i} \varepsilon_{i}$, where $\varepsilon_{i}$ approximates $\Delta L_{i}$ via a "first jump" approximation to the Lévy process.

Based on this setup, we can write down a pseudo-log-likelihood function for $Y_{1}, Y_{2}, \ldots, Y_{N}$ as in Eq. (13) of Appendix A, and maximise it to get PMLEs of $(\beta, \eta, \varphi)$. The extra regression on $\sigma_{i}^{2}$ in Eq. (1) then represents the relation between excess return and past volatility, as in Merton's (1980) formulation, with volatility modelled by a COGARCH process. If the time intervals are small enough and numerous enough, we can expect the fitted discretised model to closely approximate the underlying COGARCH. Note that one can estimate the COGARCH parameters together with the regression parameters $\lambda_{1}$ and $\lambda_{2}$ in one stage. Standard errors for all estimates can be calculated using the Hessian matrix of the likelihood function at the estimated maximum. For more details see our Appendix A.

\subsection{Accounting for trading days by time transformation}

That returns have day-of-the-week "seasonalities" is well established in the Finance literature (French, 1980; Gibbons and Hess, 1981; Erickson et al., 1997). Flannery and Protopapadakis (2002) find day of the week effects in both the mean and variance equations using GARCH $(1,1)$ in their analysis of daily returns and their sensitivity to macroeconomic announcements using data from 1980 to 1996. Therefore, in our analyses, which cover a long time span, it is incumbent upon us to take daily seasonalities effects into account. To this end we introduce exogenous indicator variables. Their influence on the volatility will be captured by switching over to a virtual time scale which we call the volatility time scale. Roughly speaking, this scale measures the volatility pattern of certain weekdays with respect to a day with average volatility. Following a series of analyses with different indicator variables for weekdays and months, we found it necessary to use only MONDAY and FRIDAY variables as being the significant ones for our data. ${ }^{17}$ In the remainder of this section, for simplicity we confine our discussion to these two covariates.

Via the virtual time scale transformation, the PML method simultaneously rescales the physical time axis and estimates the COGARCH parameters. To be precise, we replace $\Delta t_{i}$ in Eqs. (10) and (11) by

$$
\Delta \tau_{i}:=\left(3 h_{\mathrm{MON}} I_{\mathrm{MON}}(i)+I_{\mathrm{TUE}}(i)+I_{\mathrm{WED}}(i)+I_{\mathrm{THU}}(i)+h_{\mathrm{FRI}} I_{\mathrm{FRI}}(i)\right) \bar{\Delta}
$$

where $h_{\mathrm{MON}}$ and $h_{\mathrm{FRI}}$ are parameters to be estimated, and the constant $\bar{\Delta}$ serves as a basic time unit, which we set to $1 / 365.25$. The exogenous variable $I_{\text {MoN }}$ takes values 1 or 0 , to indicate whether or not the return under consideration is based on a Monday's close of trading price. The Monday return is the difference between Friday's closing price and Monday's closing price (after adjusting for dividends etc.). Therefore, $h_{\text {MON }}$ captures any weekend effect. We similarly adjust for $I_{\text {FRI }}$.

For weekly returns, we replace $\Delta t_{i}$ in Eqs. (10) and (11) by

$$
\Delta \tau_{i}:=\left(h_{\mathrm{WEEK} 1} I_{\mathrm{WEEK} 1}(i)+I_{\mathrm{WEEK} 2-52}(i)\right) \bar{\Delta},
$$

where $h_{\text {WEEK1 }}$ is treated as a parameter to be estimated; the basic time unit $\bar{\Delta}$ is set to $1 / 52.18$ in this case (since the average length of a year in weeks is about 52.18), and $I_{\text {WEEK1 }}$ and $I_{\text {WEEK2 - } 52}$ indicate whether the weekly return under consideration was observed over the first (complete) week of the year or not. ${ }^{18}$

For monthly returns, we replace $\Delta t_{i}$ in Eqs. (10) and (11) by

$$
\Delta \tau_{i}:=\left(h_{\mathrm{JAN}} I_{\mathrm{JAN}}(i)+I_{\mathrm{FEB}}(i)+I_{\mathrm{MAR}}(i)+\ldots+I_{\mathrm{NOV}}(i)+I_{\mathrm{DEC}}(i)\right) \bar{\Delta}
$$

where $h_{\mathrm{JAN}}$ is treated as a parameter to be estimated; the basic time unit $\bar{\Delta}$ is set to $1 / 12$ in this case, and $I_{\mathrm{JAN}}, \ldots, I_{\mathrm{DEC}}$ indicate the month in which the return under consideration was observed. ${ }^{19}$

\section{Data}

Our main interest is in analysing daily returns of the CRSP (Center for Research in Security Prices) value-weighted index of the returns of stocks listed in the US market (we denote this index as the VWI). Additionally, we analyse the CRSP equally weighted index (which we refer to as the EWI). For both indices, we have 13,844 daily observations in the whole period from January 1,1953

\footnotetext{
${ }^{17}$ We do not utilize indicator variables for discontinuities in trading due to regular holidays in the analysis presented in this paper. Given the period covered by the data, it is problematic to distinguish holidays from "surprise" breaks in the data (for example, the trading halt brought about by the September 11 incident). The COGARCH specification is designed to allow valid inference about the relationship we seek to model in the presence of such breaks. We believe that the GOGARCH methodology demonstrated in this paper might be useful in future analyses of holiday effects in analyses like that presented in Flannery and Protopapadakis (2002).

${ }^{18}$ Other variations of the time scale, e.g. for the last week in December, the second or third week in January, turned out not to be significant at the $5 \%$ level for our data.

${ }_{19}$ Other variations of the time scale, e.g. for December, turned out not to be significant at the $5 \%$ level for our data.
} 
to December 31,2007 . The daily returns $r_{i}, i=1, \ldots, n$, are considered as observations on the random variables $R_{i}$ in Eq. (1), over the period January 1,1927, to December 31, 2007. We divide this into two subperiods, the second period starting on January 1, 1953, to accommodate a possible major structural break in the data; the weekly pattern of trading, which plays an important role in our analyses, changed significantly when Saturday trading was abandoned in $1952 .{ }^{20}$ The data was accumulated into weekly and monthly returns for the period 1927-2007, to provide subsidiary data sets for an additional analysis.

Although describing the same market, there are significant differences between the two indices, as one can see also from simple descriptive statistics. Whereas the VWI daily returns have a mean of 0.000458 and standard deviation 0.008484 , the EWI returns have a mean of 0.000771 and a standard deviation of 0.007073 . The difference between these indices seems even stronger if one counts the days when the VWI and the EWI have different signs: out of the 13,844 observations, the VWI showed a negative return on 1471 occasions, while the EWI return was positive. Interestingly, the opposite (VWI return positive, EWI return negative) occurred only twice.

We will refer to the four different data sets, namely, daily returns 1953-2007, daily returns 1927-1952, weekly returns 1927-2007 and monthly returns 1927-2007, by the intuitive notations [D53], [D27], [W27] and [M27] respectively. Sometimes we extend this notation, e.g. to [D27]-VWI or [D27]-EWI, to specify exactly which data set is being used. We do not analyse weekly or monthly data for the shorter period beginning in 1953 in order to improve statistical reliability of our results (even for the longer period from 1927 to 2007 we have 4224 weekly and only 972 monthly observations). For the daily data, we focus on [D53] because of the structural break and use [D27] to confirm the robustness of the inferences we make about Merton's proposition. (recall that Saturday was a trading day before 1953).

To compute excess returns we obtained the risk-free rates provided by Ken French. ${ }^{21}$ These data are well known from Fama and French's seminal work in asset pricing (for example, Fama and French, 1993).

\section{Results and interpretation}

\subsection{Results for the COGARCH based models - daily data}

The pseudo-maximum likelihood estimates together with estimated standard errors for all model parameters can be found in Table 1.

Recall that we only work with the data sets [D53] and [D27] for these models. To begin our analysis, we compare the nested pairs COGARCH (U)-COGARCH (V); COGARCH (V)-asyCOGARCH (V); COGARCH (U)-asyCOGARCH (U); asyCOGARCH (U)-asyCOGARCH (V); using AIC and BIC. Both measures indicate that for all four data sets, [D53]-VWI, [D27]-VWI, [D53]-EWI and [D27]-EWI, the model fit is significantly improved, when the effects for Monday and Friday are taken into account: COGARCH (V) is preferred to COGARCH (U). The comparison of asyCOGARCH (V) to COGARCH (V) indicates that asyCOGARCH (V) is to be preferred. Both model fit criteria, AIC and BIC, punish models with a higher complexity. Nevertheless, although COGARCH (V) has two additional parameters and asyCOGARCH $(\mathrm{V})$ has three additional parameters, over COGARCH (U), the most complex (asymmetric) model is considered to be significantly superior to the competing COGARCH specifications.

Examining the results for the asyCOGARCH (V) model reported in Table 1, we find that the estimate of $\lambda_{2}$ for [D53]-VWI is positive, as hypothesised, but it is not statistically significantly different from 0 . Merton's hypothesised positive relationship of returns to risk is therefore not significantly supported by this analysis. The estimate of $\lambda_{2}$ for [D53]-VWI obtained using COGARCH $(\mathrm{V})$ is, by way of contrast to the estimate produced using asyCOGARCH (V) (the preferred model), both positive and statistically significant. This highlights the importance of considering asymmetry in analysing the relationship of return to risk. Note that $\lambda_{2}$ for [D53-VWI] using asyCOGARCH (U) is also positive and statistically significant; Merton's hypothesis would be supported by our data if we ignored daily seasonalities. For [D53-EWI], asyCOGARCH (V) is also the preferred model. ${ }^{22}$ Here it also produces a statistically insignificant estimate for $\lambda_{2}$, and, again, ignoring daily seasonalities while accounting for asymmetry when estimating asyCOGARCH (U) results in a statistically significant, positive, estimate of $\lambda_{2}$.

To assess the quality of the model fit, Fig. 1 shows the values of the scaled residuals, $R_{i}-r_{f, i}-\hat{\lambda}_{1}-\hat{\lambda}_{2} \hat{\sigma}_{i}^{2}$, for the VWI, with estimates based on the asyCOGARCH (V) model for the period 1953-2007, and the corresponding estimated annualised volatilities. For the EWI we obtained a very similar picture (which we omit here).

We repeated the analysis on the second dataset of daily returns between 1927 and 1951, the period when trading took place on Saturdays (in contrast to the period from 1953 to 2007) and also report those results in Table 1. Here asyCOGARCH (V) is, again, the optimal model for both the VWI and the EWI. In no instance is the estimate of $\lambda_{2}$ statistically significant. In contrast to the examination of the later period, in this earlier period we do not find that ignoring day-of-the-week effects (using asyCOGARCH (U)) results in statistically significant estimates of $\lambda_{2}$. On balance, the weight of evidence from the analysis thus presented is not supportive of Merton's proposition: with a realistic model allowing for an asymmetric relationship between risk and return, and a comprehensive analysis, we are not able to detect a statistically significant positive relationship between risk and return.

\footnotetext{
20 We omit 1952 from our analyses to ensure that the two periods are clearly differentiated.

21 See http://mba.tuck.dartmouth.edu/pages/faculty/ken.french/index.html. Both daily and monthly rates are available but inspection of the data revealed that daily rates are calculated as the monthly risk free rates divided by the precise number of trading days in that month. Our daily data is derived thereby from French's monthly data.

22 Our findings for EWI might be affected by autocorrelation induced by thin-trading (Campbell, Lo and MacKinlay, 1997, pages 92 to 94). To consider any possible effect of thin-trading, we analysed [D53]-EWI and [D27]-EWI by augmenting asyCOGARCH(V) with the lagged excess return of the EWI. This additional analysis does not alter the inferences we made about Merton's hypothesis on the basis of the results reported in Table 1.
} 
Table 1

COGARCH model: Estimates for the regression parameters $\lambda_{1}$ and $\lambda_{2}$, for the covariates MONDAY, FRIDAY and SATURDAY, and for the three (four) COGARCH parameters $\beta, \eta, \varphi\left(\varphi^{+} / \varphi^{-}\right)$. Data sets [D53] (13,844 observations) and [D27] (7357 observations), both for VWI and EWI. Estimated standard errors in parentheses.

\begin{tabular}{|c|c|c|c|c|c|c|c|c|c|}
\hline \multicolumn{10}{|c|}{ VWI: 1953-2007 (daily) } \\
\hline Model & $\beta$ & $\eta$ & $\varphi\left(\varphi^{+} / \varphi^{-}\right)$ & $h_{\mathrm{MON}}$ & $h_{\mathrm{FRI}}$ & $\lambda_{1} \cdot 10^{3}$ & $\lambda_{2}$ & AIC & $\mathrm{BIC}$ \\
\hline asyV & $0.114(0.007)$ & $29.49(1.212)$ & $10.40(0.640) / 39.35(1.438)$ & $0.448(0.013)$ & $0.972(0.029)$ & $0.371(0.080)$ & $0.542(0.844)$ & $-97,253.6$ & $-97,193.3$ \\
\hline asyU & $0.164(0.009)$ & $35.34(1.347)$ & $11.99(0.672) / 45.93(1.541)$ & & & $0.161(0.069)$ & $2.852(0.891)$ & $-97,142.1$ & $-97,096.9$ \\
\hline V & $0.118(0.009)$ & $33.75(1.428)$ & $30.40(1.339)$ & $0.447(0.013)$ & $0.965(0.029)$ & $0.374(0.083)$ & $3.262(1.421)$ & $-97,053.6$ & $-97,000.8$ \\
\hline $\mathrm{U}$ & $0.156(0.007)$ & $39.24(1.194)$ & $34.79(1.062)$ & & & $0.215(0.085)$ & $5.440(1.430)$ & $-96,931.0$ & $-96,893.3$ \\
\hline
\end{tabular}

VWI: 1927-.1951 (daily)

\begin{tabular}{|c|c|c|c|c|c|c|c|c|c|}
\hline Model & $\beta$ & $\eta$ & $\varphi\left(\varphi^{+} / \varphi^{-}\right)$ & $h_{\mathrm{MON}}$ & $h_{\mathrm{SAT}}$ & $\lambda_{1} \cdot 10^{3}$ & $\lambda_{2}$ & AIC & $\mathrm{BIC}$ \\
\hline asyV & $0.240(0.016)$ & $48.66(1.882)$ & $27.45(1.174) / 67.69(2.005)$ & $0.586(0.016)$ & $0.395(0.012)$ & $0.641(0.091)$ & $0.096(1.304)$ & $-48,045.0$ & $-47,989.8$ \\
\hline asyU & $0.247(0.015)$ & $45.96(1.858)$ & $22.22(1.093) / 64.77(1.914)$ & & & $0.578(0.083)$ & $0.564(1.427)$ & $-47,560.3$ & $-47,518.8$ \\
\hline V & $0.268(0.018)$ & $47.92(1.714)$ & $50.63(1.876)$ & $0.592(0.016)$ & $0.396(0.012)$ & $0.897(0.104)$ & $0.149(1.383)$ & $-47,935.3$ & $-47,887.0$ \\
\hline $\mathrm{U}$ & $0.277(0.020)$ & $46.24(1.592)$ & $49.37(1.622)$ & & & $0.606(0.083)$ & $1.771(1.420)$ & $-47,425.5$ & $-47,391.0$ \\
\hline
\end{tabular}

EWI: 1953-2007 (daily)

\begin{tabular}{|c|c|c|c|c|c|c|c|c|c|}
\hline Model & $\beta$ & $\eta$ & $\varphi\left(\varphi^{+} / \varphi^{-}\right)$ & $h_{\mathrm{MON}}$ & $h_{\mathrm{FRI}}$ & $\lambda_{1} \cdot 10^{3}$ & $\lambda_{2}$ & AIC & BIC \\
\hline asyV & $0.305(0.012)$ & $86.21(3.862)$ & $52.25(1.473) / 91.96(3.226)$ & $0.491(0.014)$ & $0.901(0.028)$ & $0.968(0.064)$ & $0.882(0.939)$ & $-103,069.5$ & $-103,009.2$ \\
\hline asyU & $0.406(0.019)$ & $101.1(3.989)$ & $56.70(1.512) / 110.8(3.509)$ & & & $0.826(0.061)$ & $2.387(0.972)$ & $-102,874.1$ & $-102,828.9$ \\
\hline V & $0.328(0.023)$ & $94.06(4.304)$ & $85.90(4.421)$ & $0.505(0.015)$ & $0.882(0.027)$ & $1.095(0.070)$ & $1.010(1.573)$ & $-103,000.4$ & $-102,947.6$ \\
\hline $\mathrm{U}$ & $0.428(0.029)$ & $109.1(4.366)$ & $98.27(4.811)$ & & & $0.754(0.070)$ & $5.316(1.514)$ & $-102,773.1$ & $-102,735.4$ \\
\hline
\end{tabular}

EWI: 1927-1951 (daily)

\begin{tabular}{|c|c|c|c|c|c|c|c|c|c|}
\hline Model & $\beta$ & $\eta$ & $\varphi\left(\varphi^{+} / \varphi^{-}\right)$ & $h_{\mathrm{MON}}$ & $h_{\mathrm{SAT}}$ & $\lambda_{1} \cdot 10^{3}$ & $\lambda_{2}$ & AIC & $\mathrm{BIC}$ \\
\hline asyV & $0.524(0.030)$ & $69.25(2.476)$ & $49.39(2.280) / 96.15(3.553)$ & $0.623(0.017)$ & $0.407(0.013)$ & $1.216(0.158)$ & $0.751(1.184)$ & $-45,964.9$ & $-45,909.7$ \\
\hline asyU & $0.574(0.031)$ & $68.37(2.415)$ & $43.51(2.234) / 96.39(3.570)$ & & & $0.808(0.141)$ & $2.293(1.263)$ & $-45,465.8$ & $-45,424.4$ \\
\hline V & $0.625(0.037)$ & $68.04(2.794)$ & $71.03(2.975)$ & $0.629(0.017)$ & $0.412(0.013)$ & $1.300(0.160)$ & $2.809(1.295)$ & $-45,870.5$ & $-45,822.2$ \\
\hline $\mathrm{U}$ & $0.700(0.046)$ & $69.23(2.805)$ & $72.78(2.985)$ & & & $0.712(0.136)$ & $5.111(1.371)$ & $-45,351.5$ & $-45,316.9$ \\
\hline
\end{tabular}

\subsubsection{Weekend effects}

In the asyCOGARCH (V) models for [D53] (the 1953-2007 daily data), the ML estimates for the exogenous variable MONDAY are 0.448 and 0.491 for the VWI and EWI data set, respectively, and are significantly positive, consistent with literature confirming daily seasonalities (for example, French, 1980; Gibbons and Hess, 1981; Erickson et al., 1997; Flannery and Protopapadakis, 2002). Since the log-returns computed at close of trading on Monday each week are based on the market information of 3 days, the time between close of trading on Friday and close of trading on Monday corresponds to $3 \times 0.448=1.344$ volatility days on our virtual time scale in the VWI data, and to $3 \times 0.491=1.473$ volatility days in the EWI data (cf. Eq. (6) in Section 2.2 ). Therefore, we can conclude that neither ignoring the weekend nor counting it as two days would constitute a satisfying approximation for our purposes. Rather, a weekend should be counted as about 0.3 to 0.5 regular trading days. The difference between the VWI and EWI estimates for MONDAY is not significant, nor is it for the FRIDAY estimates, which are 0.972 and 0.901 for the VWI and EWI data, respectively. Only for the equally weighted index is the coefficient significantly smaller than 1 , indicating a slightly smaller volatility on Fridays compared to other weekdays, for this index.

For [D27] (daily data from 1927 to 1951) analysed by the asyCOGARCH (V) model, the ML estimates for the exogenous variable SATURDAY are 0.395 and 0.407 for the VWI and EWI data set, respectively, again significantly different from 0 or 1 . This means that volatility on Saturdays was, in general, much smaller than on the other trading days. The estimates for the variable MONDAY for this period were 0.586 and 0.623 respectively, reflecting the period from Saturday close to Monday close, i.e. two days. Hence, Sunday was equivalent to about 0.2 trading days in this period, in terms of volatility.

\subsubsection{Equity risk premia estimates}

To compute an estimate of the long-term risk premium from the asyCOGARCH (V) models for the 1953-2007 daily data, we use estimates $\overline{\sigma_{i}^{2}}$ and $\overline{\sigma_{i}}$ for the average variance and volatility. Since (as with any weighted regression) the residuals do not exactly average to 0 , we also take their ( small) bias into account. ${ }^{23}$ The estimate $\hat{p}$ for the long-term risk premium is then computed as

$$
\hat{p}=\hat{\lambda}_{1}+\hat{\lambda}_{2} \overline{\sigma_{i}^{2}}+\overline{\sigma_{i}} \overline{\varepsilon_{i}}
$$

\footnotetext{
${ }^{23}$ This effect is due to the PML estimation procedure and should not be neglected, cf. Maller and Müller (2010).
} 
Excess returns after subtracting estimated regression components

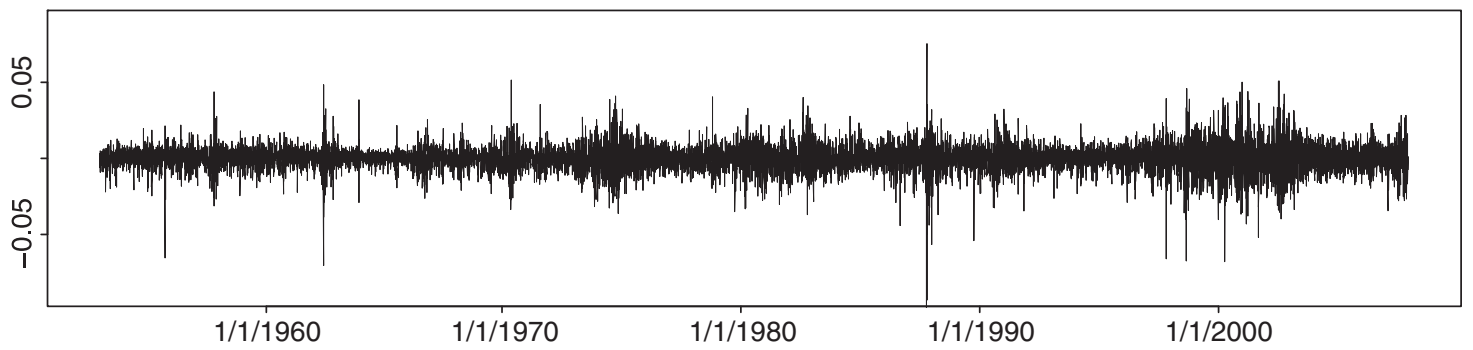

Corresponding estimated annualised volatilities

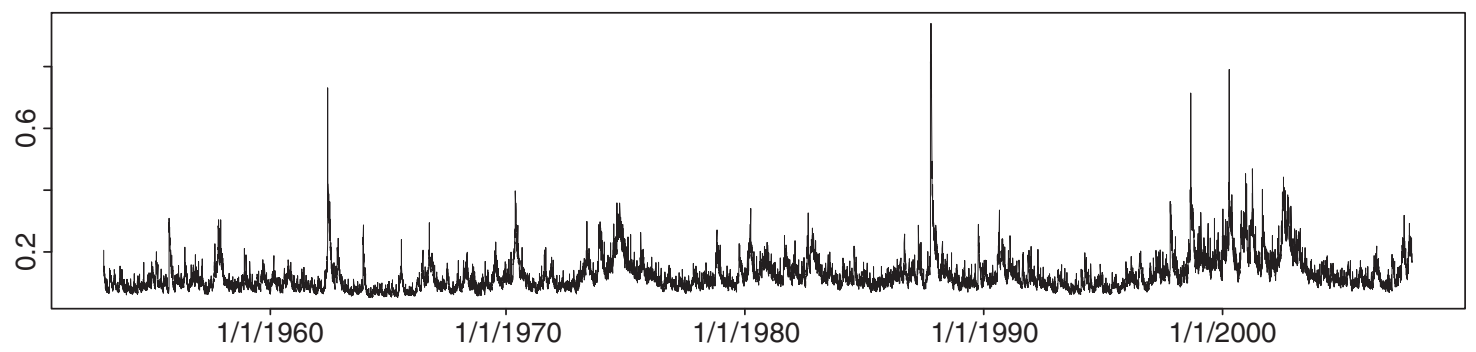

Fig. 1. Top: Values of $R_{i}-r_{f, i}-\hat{\lambda}_{1}-\hat{\lambda}_{2} \hat{\sigma}_{i}^{2}$ for the VWI, with estimates based on the period 1953-2007. Bottom: Corresponding estimated annualised volatilities.

Fitting asyCOGARCH(V) to data set D53-VWI, we get the estimate $\hat{p}=7.17 \%$ p.a. (Table 2 ). It is instructive to compare this value with those reported in Table 1 of Welch (2000, page 504), who summarises estimates of the historical stock market and equity premia from a variety of sources. Our estimate for the period $1953-2007$ is close to Shiller's estimate of $\hat{p}=6.9 \%$ for the geometric mean, and $\hat{p}=8.2 \%$ p.a. for the arithmetic mean, for the similar period 1949-1998; but our study period includes the tech boom, and subsequent bust, that continued after Shiller's fifty year period, as well as the returns enjoyed before the global financial crisis began towards the end of 2007. Furthermore, estimates of the equity risk premium produced for this dataset using the other variants of COGARCH we analyse, asyCOGARCH(U), COGARCH (V) and COGARCH $(\mathrm{U})$ are $7.75 \%, 7.78 \%$ and $7.38 \%$ respectively. All of these estimates are within the range reported by Welch. Therefore, our inferences regarding the equity risk premium are robust as to whether we control for daily seasonalities and asymmetry or not.

The estimated excess return for the EWI estimated using asyCOGARCH (V) is $\hat{p}=18 \%$ p.a., and for COGARCH (U) it is $16.83 \%$. These may appear high, but in fact the EWI had an average return of $19.39 \%$ per year over the years $1953-2007 .{ }^{24}$ This figure is, furthermore, in keeping with the upper end of financial economists' optimistic forecasts for the long run equity premium. Welch surveyed financial economists' expectations and found that the optimistic projection of the equity premium over thirty years is between $11 \%$ and $13 \%$, the optimistic projection over the ten-year horizon was around $15 \%$ and for the five-year horizon was around $20 \%$ (Welch, 2000, page 515).

Although the VWI includes all the stocks listed in America, it is dominated by those having the largest market capitalization (that is, large stocks). The EWI, in contrast, will reflect stocks with smaller market capitalizations (including "penny stocks") which are believed to generate generous returns (Bhardwaj and Brooks, 1992; Brockman and Michayluk, 1997). That small firms earn a premium is well-known in the literature and there are theoretical arguments as to why this should be the case (see Banz, 1981; Keim, 1983; Brown et al., 1983; Reinganum and Shapiro, 1987; James and Edmister, 1983; Berk, 1995). ${ }^{25}$

Estimates of the equity risk-premium produced by all the COGARCH models for the VWI for the period 1927 to 1951 are similar, ranging from $8.23 \%$ to $8.68 \%$, in keeping with the COGARCH estimates for the latter period and also with the estimates of the equity risk-premium provided in Welch (2000, page 504). In contrast, the estimated equity risk premium produced by the four COGARCH models for the EWI for 1927 to 1951 are higher than those produced for the latter period, ranging from $25.65 \%$ to $26.68 \%$.

\subsection{Results for GARCH, QGARCH, GJRGARCH and TGARCH based models}

In practice, econometricians are faced with a bewildering array of conditional volatility models, often non-nested. There is no easy way of choosing an appropriate one. We have argued that, within the class of stochastic volatility models encompassed by the GARCH

\footnotetext{
${ }^{24}$ We obtain this figure simply by averaging the daily returns.

25 Is the higher expected return for the EWI simply a function of higher expected risk? Although the statistical insignificance of $\widehat{\aleph}_{2}$ is contrary to what might be expected given Merton's proposition, further consideration of the data in instructive. The average return of the EWI is 0.000771 per day, almost twice that of the VWI, at 0.000458 . The standard deviation of daily returns for the EWI, however, is less than that of the VWI $(0.007073$ for the EWI vs. 0.008484 for the VWI). This appears to contradict expectations concerning the return to risk relationship. Merton's hypothesis, however, relates expected risk to returns, and this is where consideration of the COGARCH parameters is potentially helpful. The long run estimate of volatility, computed using $\beta /(\eta-\varphi)$, is higher for the EWI $(0.0403)$ than for the VWI (0.0345). Therefore, when we consider investors' expectations, the higher returns for EWI are consistent with the higher expected risk borne by investors exposed to that index.
} 
Table 2

Estimated risk premia and volatilities: Minimal estimated annualised volatilities in \% $\left(\sigma_{\min }\right)$, maximal estimated annualised volatilities in \% $\left(\sigma_{\text {max }}\right)$, and average of estimated annualised volatilities in $\%\left(\sigma_{\text {mean }}=\overline{\sigma_{i}}\right)$. Fifth and ninth column: Average excess return premium in \% per year.

\begin{tabular}{|c|c|c|c|c|c|c|c|c|}
\hline \multirow[t]{2}{*}{ Model } & $\sigma_{\min }$ & $\sigma_{\max }$ & $\sigma_{\text {mean }}$ & Premium & $\sigma_{\min }$ & $\sigma_{\max }$ & $\sigma_{\text {mean }}$ & Premium \\
\hline & \multicolumn{4}{|c|}{ VWI: 1953-2007 (daily) } & \multicolumn{4}{|c|}{ EWI: 1953-2007 (daily) } \\
\hline COGARCH (asyV) & 5.47 & 93.99 & 12.27 & 7.17 & 4.88 & 89.58 & 10.03 & 18.00 \\
\hline COGARCH (asyU) & 5.97 & 97.22 & 12.26 & 7.75 & 5.36 & 98.04 & 10.06 & 17.12 \\
\hline COGARCH (V) & 5.38 & 87.50 & 12.28 & 7.78 & 4.83 & 90.72 & 10.06 & 17.13 \\
\hline COGARCH (U) & 5.84 & 87.35 & 12.29 & 7.38 & 5.34 & 97.23 & 10.08 & 16.83 \\
\hline GARCH & 5.72 & 89.16 & 12.51 & 7.58 & 5.25 & 96.25 & 11.29 & 16.10 \\
\hline QGARCH & 5.70 & 85.11 & 12.21 & 8.37 & 5.24 & 90.04 & 11.18 & 18.38 \\
\hline GJRGARCH & 6.12 & 101.54 & 12.49 & 7.36 & 5.48 & 101.98 & 11.29 & 17.44 \\
\hline \multirow[t]{2}{*}{ Model } & $\sigma_{\min }$ & $\sigma_{\max }$ & $\sigma_{\text {mean }}$ & Premium & $\sigma_{\min }$ & $\sigma_{\max }$ & $\sigma_{\text {mean }}$ & Premium \\
\hline & \multicolumn{4}{|c|}{ VWI: 1927-1951 (daily) } & \multicolumn{4}{|c|}{ EWI: 1927-1951 (daily) } \\
\hline COGARCH (asyV) & 4.32 & 179.65 & 20.71 & 8.53 & 5.22 & 219.53 & 23.61 & 26.12 \\
\hline COGARCH (asyU) & 6.85 & 113.40 & 20.66 & 8.23 & 8.02 & 134.75 & 23.65 & 25.65 \\
\hline COGARCH (V) & 4.44 & 167.96 & 20.30 & 8.58 & 5.58 & 197.86 & 22.85 & 26.13 \\
\hline COGARCH (U) & 7.15 & 107.14 & 20.36 & 8.68 & 8.55 & 128.55 & 23.00 & 26.68 \\
\hline \multirow[t]{2}{*}{ Model } & $\sigma_{\min }$ & $\sigma_{\max }$ & $\sigma_{\text {mean }}$ & Premium & $\sigma_{\min }$ & $\sigma_{\max }$ & $\sigma_{\text {mean }}$ & Premium \\
\hline & \multicolumn{4}{|c|}{ VWI: 1927-2007 (weekly) } & \multicolumn{4}{|c|}{ EWI: 1927-2007 (weekly) } \\
\hline COGARCH (asyV) & 6.46 & 62.97 & 17.41 & 7.33 & 7.15 & 94.81 & 20.35 & 19.34 \\
\hline COGARCH (asyU) & 7.26 & 63.43 & 17.43 & 7.27 & 7.11 & 95.06 & 20.35 & 18.98 \\
\hline COGARCH (V) & 6.26 & 63.75 & 17.20 & 7.47 & 7.40 & 86.59 & 19.69 & 19.61 \\
\hline COGARCH $(U)$ & 7.15 & 64.12 & 17.22 & 7.46 & 7.40 & 86.44 & 19.69 & 19.61 \\
\hline \multirow[t]{2}{*}{ Model } & $\sigma_{\min }$ & $\sigma_{\max }$ & $\sigma_{\text {mean }}$ & Premium & $\sigma_{\min }$ & $\sigma_{\max }$ & $\sigma_{\text {mean }}$ & Premium \\
\hline & \multicolumn{4}{|c|}{ VWI: 1927-2007 (monthly) } & \multicolumn{4}{|c|}{ EWI: 1927-2007 (monthly) } \\
\hline COGARCH (asyV) & 7.41 & 60.90 & 18.60 & 8.16 & 10.44 & 79.39 & 23.89 & 20.28 \\
\hline COGARCH (asyU) & 9.10 & 60.51 & 18.66 & 8.11 & 11.67 & 78.31 & 23.93 & 20.56 \\
\hline COGARCH (V) & 7.38 & 61.42 & 16.79 & 7.87 & 10.24 & 88.02 & 21.58 & 19.45 \\
\hline COGARCH (U) & 9.17 & 60.97 & 16.85 & 7.85 & 11.85 & 87.13 & 21.61 & 19.50 \\
\hline GARCH & 4.34 & 63.95 & 18.80 & 8.02 & 5.08 & 89.47 & 24.38 & 20.28 \\
\hline QGARCH & 4.84 & 62.06 & 18.66 & 8.14 & 5.41 & 88.17 & 24.24 & 20.49 \\
\hline GJRGARCH & 4.57 & 58.70 & 18.68 & 7.95 & 5.18 & 79.90 & 24.09 & 20.40 \\
\hline
\end{tabular}

paradigm, a COGARCH specification is appropriate due to its explicit recognition of an important feature of the data - unequal spacing. But COGARCH is just one among a range of similar competing GARCH-based models and, for comparison, we also fitted some other commonly used discrete time GARCH models to the data. This practice ignores the unequal spacing of the observations; however, it is the kind of approximation that had to be made before COGARCH was available. We briefly describe the discrete time GARCH-based models we used. First, the usual GARCH(1,1) model was fitted. We also used a QGARCH(1,1), a GJRGARCH(1,1) (Glosten-Jagannathan-Runkle-GARCH), and a TGARCH(1,1) model. ${ }^{26}$

To specify the discrete time models precisely, abbreviate $Y_{i}:=R_{i}-r_{f, i}$ and $Z_{i}=Y_{i}-\left(\lambda_{1}+\lambda_{2} \sigma_{i}^{2}\right)$. Then these models all follow the equation $Z_{i}=\sigma_{i} \varepsilon_{i}$, where the volatility process is given by one of the following equations:

$$
\begin{array}{ll}
\text { GARCH : } & \sigma_{i+1}^{2}=a_{\mathrm{G}}+b_{\mathrm{G}} \sigma_{i}^{2}+c_{\mathrm{G}} Z_{i}^{2} \\
\text { QGARCH : } & \sigma_{i+1}^{2}=a_{\mathrm{Q}}+b_{\mathrm{Q}} \sigma_{i}^{2}+c_{\mathrm{Q}} Z_{i}^{2}+d_{\mathrm{Q}} Z_{i} \\
\text { GJRGARCH : } & \sigma_{i+1}^{2}=a_{\mathrm{GJR}}+b_{\mathrm{GJR}} \sigma_{i}^{2}+c_{\mathrm{GJR}} Z_{i}^{2}+e_{\mathrm{GJR}} Z_{i}^{2} I_{i}
\end{array}
$$

In the GJRGARCH model the variable $I_{i}$ takes the values $I_{i}=1$ if $Z_{i}<0$ and $I_{i}=0$ if $Z_{i} \geq 0$ and, hence, allows for asymmetry in the distribution of the returns.

We present the estimates for the GARCH, QGARCH and GJRGARCH based models for both the VWI and EWI datasets, daily (beginning in 1953) and monthly data (beginning in 1927), in Table 3. As previously, we compare nested models by AIC and BIC. ${ }^{27}$

\footnotetext{
26 Since it turned out that the TGARCH showed a significantly worse model fit than the plain GARCH model (as judged by AIC and BIC), we omit in the following all results for the TGARCH based models and just report the results for the other three GARCH models.

27 Note that only the following pairs are nested (smaller model first): GARCH-QGARCH, GARCH-GJRGARCH, GARCH-TGARCH.
} 
Table 3

Discrete time GARCH models: Parameter estimates with standard errors for GARCH, QGARCH, and GJRGARCH models fitted to VWI and EWI indices. Data sets [D53] (13,844 observations) and [M27] (972 observations).

\begin{tabular}{|c|c|c|c|c|c|c|c|c|c|c|}
\hline \multicolumn{11}{|c|}{ VWI: 1953-2007 (daily) } \\
\hline Model & $a \cdot 10^{6}$ & $b$ & c & & $d \cdot 10^{3}$ & $e \cdot 10^{3}$ & $\lambda_{1} \cdot 10^{3}$ & $\lambda_{2}$ & AIC & BIC \\
\hline GARCH & $1.085(0.063)$ & $0.898(0.004)$ & 0.0 & $88(0.004)$ & & & $0.215(0.080)$ & $5.368(1.672)$ & $-96,932.5$ & $-96,894.8$ \\
\hline QGARCH & $1.825(0.192)$ & $0.889(0.006)$ & 0.0 & $82(0.007)$ & $-0.533(0.047)$ & & $0.164(0.094)$ & $3.439(1.563)$ & $-97,136.7$ & $-97,091.5$ \\
\hline GJRGARCH & $1.263(0.091)$ & $0.903(0.004)$ & 0.0 & $29(0.005)$ & & $94.532(6.582)$ & $0.267(0.083)$ & $1.246(1.376)$ & $-97,172.0$ & $-97,126.7$ \\
\hline \multicolumn{11}{|c|}{ VWI: 1927-2007 (monthly) } \\
\hline Model & $a \cdot 10^{6}$ & $b$ & & $c$ & $d \cdot 10^{3}$ & $e \cdot 10^{3}$ & $\lambda_{1} \cdot 10^{3}$ & $\lambda_{2}$ & AIC & BIC \\
\hline GARCH & $71.547(21.23$ & $0.853(0.0$ & 21) & $0.125(0.0$ & 19) & & $6.370(2.0$ & $0.789(0.86$ & -3217.7 & -3193.3 \\
\hline QGARCH & $110.836(30.93$ & $0.837(0.0$ & & $0.124(0.0$ & $-3.862(1.2$ & & $5.814(2.0$ & $0.568(0.91$ & -3224.9 & -3195.6 \\
\hline GJRGARCH & $88.597(25.60$ & $0.851(0.0$ & & $0.076(0.0$ & & $75.841(36$. & $6.342(2.1$ & $0.514(0.91$ & -3219.5 & -3190.2 \\
\hline
\end{tabular}

EWI: 1953-2007 (daily)

\begin{tabular}{|c|c|c|c|c|c|c|c|c|c|}
\hline Model & $a \cdot 10^{6}$ & $b$ & c & $d \cdot 10^{3}$ & $e \cdot 10^{3}$ & $\lambda_{1} \cdot 10^{3}$ & $\lambda_{2}$ & AIC & BIC \\
\hline GARCH & $2.840(0.195)$ & $0.740(0.010)$ & $0.206(0.012)$ & & & $0.762(0.069)$ & $5.130(1.516)$ & $-102,774.5$ & $-102,736.8$ \\
\hline QGARCH & $3.525(0.218)$ & $0.723(0.009)$ & $0.203(0.012)$ & $-0.740(0.056)$ & & $0.878(0.075)$ & $0.143(1.790)$ & $-103,010.7$ & $-102,965.5$ \\
\hline GJRGARCH & $2.998(0.197)$ & $0.742(0.007)$ & $0.109(0.010)$ & & 165.703 (13.59) & $0.872(0.072)$ & $0.132(1.643)$ & $-102,966.8$ & $-102,921.6$ \\
\hline
\end{tabular}

EWI: 1927-2007 (monthly)

\begin{tabular}{|c|c|c|c|c|c|c|c|c|c|}
\hline Model & $a \cdot 10^{6}$ & $b$ & $c$ & $d \cdot 10^{3}$ & $e \cdot 10^{3}$ & $\lambda_{1} \cdot 10^{3}$ & $\lambda_{2}$ & AIC & BIC \\
\hline GARCH & $130.523(31.41)$ & $0.844(0.021)$ & $0.131(0.021)$ & & & 7.675 (2.507) & $2.349(0.670)$ & -2764.3 & -2739.9 \\
\hline QGARCH & $160.187(42.48)$ & $0.836(0.024)$ & $0.130(0.025)$ & $-3.335(1.352)$ & & $7.458(2.566)$ & $2.152(0.705)$ & -2768.9 & -2739.6 \\
\hline GJRGARCH & $139.016(37.29)$ & $0.845(0.027)$ & $0.094(0.024)$ & & $60.130(31.40)$ & 7.207 (2.589) & $2.358(0.703)$ & -2765.8 & -2736.5 \\
\hline
\end{tabular}

For the VWI daily data, the estimates of $\hat{\lambda}_{2}$ are high and significantly positive when estimated using GARCH and QGARCH (5.368 and 3.439 respectively). The GJRGARCH model, like the asyCOGARCH models, recognises the asymmetry effect, and, in keeping with our findings from the asyCOGARCH(V) model, the estimate of $\hat{\lambda}_{2}$ for this model is less than one standard error away from zero.

Just as in Section 4.1.2 we utilized estimates of $\overline{\sigma_{i}^{2}}$ and $\overline{\sigma_{i}}$ of the average variance and volatility to estimate the long-term risk premium from the COGARCH (V) models for the 1953-2007 daily data, obtaining estimates in line with published data. We also calculated the equity risk-premium obtained after fitting GARCH, QGARCH and GJRGARCH, and reported these in Table 2. The estimates obtained from these models, both for the VWI and EWI, are consistent with those obtained using COGARCH. The estimated volatilities are also in keeping with those computed using COGARCH, save for the maximum volatility for VWI and EWI estimated using GJRCARCH (101.54 and 101.98 respectively) which are considerably higher than the others reported in the table. Therefore, as far as these estimates are concerned, there is little to distinguish between the competing models.

To further explore the volatility estimates produced by the different GARCH and COGARCH models, we conducted further analyses of cross-correlations and autocorrelations and report the results in Table 4. We only report the results for the VWI in the period 1953-2007 using daily data (as other datasets result in the same inferences being drawn). The diagonal in Table 4 reports the autocorrelations between the estimated values of $\sigma_{i}$ for lag 1 for all COGARCH and GARCH models. In the upper triangle of Table 4 we find the cross-correlations at lag 0 for all pairs of models. The cross-correlations for lag 1 are reported in the lower triangle of Table 4. All of the autocorrelations and cross-correlations appear high: there is no number below 0.9. The conclusions we reached on the basis of our consideration of Table 2 are unchanged: we still find that there is little that allows us to distinguish between competing models.

We also illustrate the estimated (annualised) volatilities from the various GARCH and COGARCH models for the years 1973 and 1974 (again using the daily returns of the VWI) in Fig. 2. While these figures seem to confirm the conclusion that it is the difficult to

Table 4

Auto- and cross-correlations of estimated volatilities: Upper triangle: cross-correlations at lag 0. Diagonal: autocorrelations at lag 1. Lower triangle: cross-correlations at lag 1.

\begin{tabular}{|c|c|c|c|c|c|c|c|}
\hline & asyV & asyU & V & $\mathrm{U}$ & GJR & $\mathrm{Q}$ & GARCH \\
\hline COGARCH (asyV) & 0.9184 & 0.9686 & 0.9818 & 0.9511 & 0.9657 & 0.9574 & 0.9511 \\
\hline COGARCH (asyU) & 0.9536 & 0.9805 & 0.9472 & 0.9802 & 0.9984 & 0.9893 & 0.9802 \\
\hline COGARCH (V) & 0.9031 & 0.9371 & 0.9147 & 0.9672 & 0.9421 & 0.9492 & 0.9672 \\
\hline COGARCH (U) & 0.9412 & 0.9657 & 0.9532 & 0.9812 & 0.9760 & 0.9834 & 0.9998 \\
\hline GJRGARCH & 0.9481 & 0.9753 & 0.9237 & 0.9522 & 0.9777 & 0.9910 & 0.9760 \\
\hline QGARCH & 0.9407 & 0.9670 & 0.9303 & 0.9589 & 0.9695 & 0.9776 & 0.9835 \\
\hline GARCH & 0.9412 & 0.9656 & 0.9531 & 0.9811 & 0.9624 & 0.9693 & 0.9812 \\
\hline
\end{tabular}



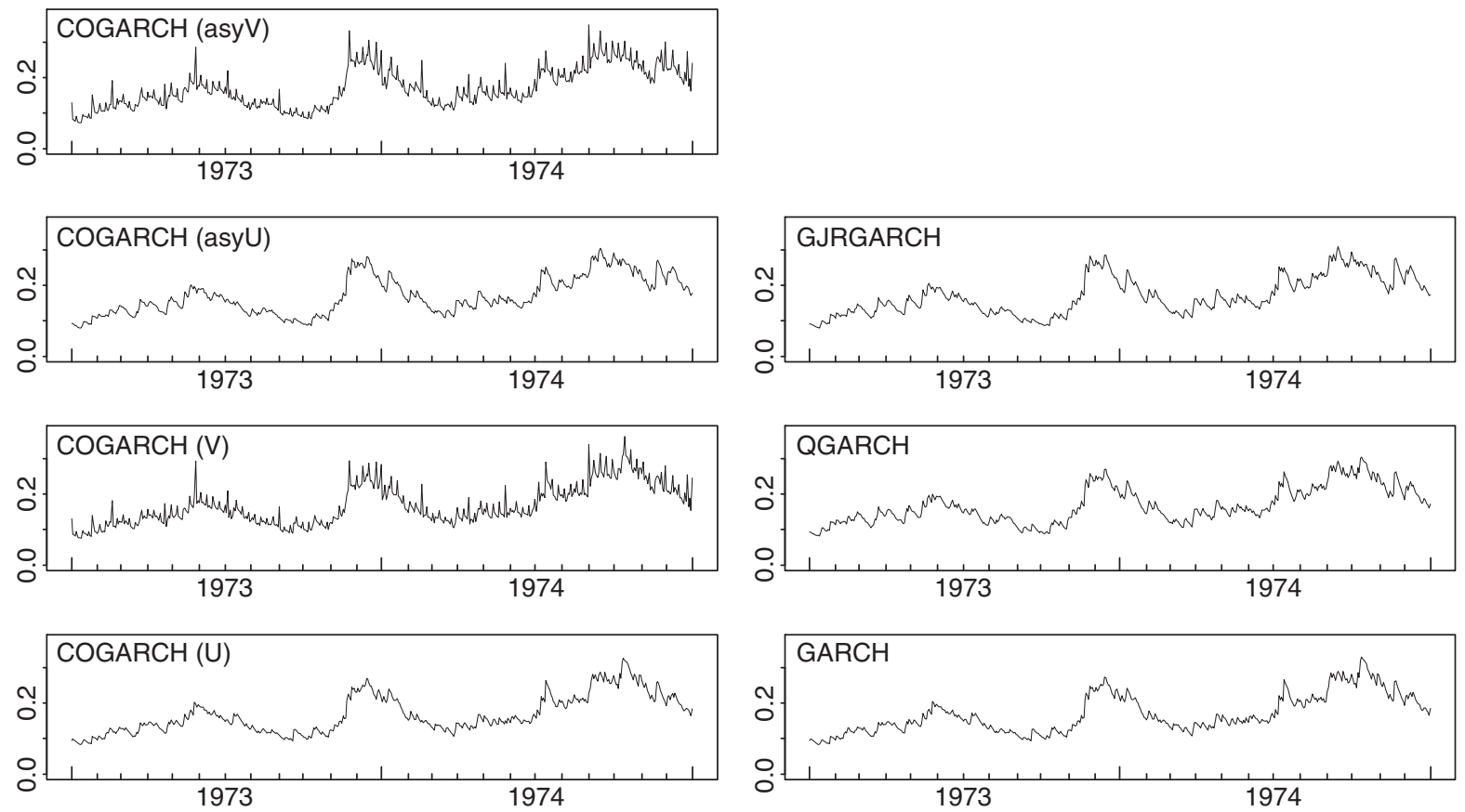

Fig. 2. Estimated annualised volatilities for the VWI, based on daily data in the period 1953-2007, for years 1973 and 1974 . Left: COGARCH models. Right: Classical GARCH models.

choose between models, the plots in Fig. 2 may begin to help us understand why the AIC and BIC select the COGARCH (asyV) model. The similarities between the pairs COGARCH (U)-GARCH and COGARCH (asyU)-GJR-GARCH are clear. Additionally, the three discrete-time GARCH models on the right-hand side of Fig. 2 also are very similar. Differences become apparent when we examine the COGARCH models. Volatility estimates vary noticeably more for the asyV and V models than the asyU and $U$ models. This effect is due to the virtual time scale. Both the asyV and V models account for the weekend; they produce volatility spikes each Monday representing the accumulated market information from Friday close to Monday close (the other models smooth over these effects). Comparing the plots for COGARCH (asyU) and COGARCH (U) one can see slight differences, which improve, as we know from the AIC and BIC, the model fit significantly.

\subsection{COGARCH (V) models for 1927-2007, weekly and monthly data}

Our use of daily data was motivated, as we have discussed, by Lundblad (2007), who demonstrates that studies of this problem may have been hampered by small samples. But asset pricing tests are typically undertaken using monthly data (see, for example, Fama and French, 1993) and Merton's seminal analysis also utilized monthly observations (Merton, 1980), ${ }^{28}$ so we also fitted the COGARCH to some lower frequency data. Given the similarities observed in the analyses of the daily data, we expect little difference between COGARCH and GARCH, QGARCH and GJRGARCH weekly and monthly analyses, also.

The COGARCH analyses of VWI and EWI for weekly and monthly data from 1927 to 2007 are in Table 5.

The estimates of the January premium, for both the VWI and EWI weekly and monthly data, are positive and statistically significant. These results are consistent with the well-known January risk-premium (Banz, 1981; Keim, 1983).

For the VWI, the analyses of the weekly and monthly data find no instance where $\hat{\lambda}_{2}$ is statistically significant. The estimates of $\hat{\lambda}_{2}$ are, in all cases, less than two standard deviations from the mean. Therefore, these analyses do not support Merton's proposal, as expected given Lundblad's (2007) findings.

For the EWI, the picture is very different. In all the analyses of the weekly and monthly data, $\hat{\lambda}_{2}$ is statistically significant. Despite the lower power of the tests, because of the smaller sample size used, we find a positive risk-return relationship.

Extrapolating these estimates to annualised equity risk-premia in Table 2 results in estimates consistent with observed values in Welch (2000, page 504). We obtain similar results when we repeat the analyses of VWI for monthly data using discrete time

\footnotetext{
28 Merton notes, however, that “...a reasonably accurate estimate of the variance rate can be obtained using daily data while the estimates for expected return taken directly from the sample will be subject to so much error as to be almost useless...in practice, the choice of an ever-shorter observation interval introduces another type of error which will 'swamp' the benefit of a shorter time interval long before the continuous time limit is reached" (Merton, 1980, page 357). Merton continues by giving the example of estimating variance using discontinuous microstructure data (which would have been unavailable to him). This, of course, is the scenario which motivates the use of COGARCH. The applicability of COGARCH to data obtained at 5 min intervals is demonstrated in Müller et al. (2009).
} 
Table 5

COGARCH model: Estimates for the regression parameters $\lambda_{1}$ and $\lambda_{2}$, for the covariates JANUARY and WEEK1, and for the three (four) COGARCH parameters $\beta, \eta, \varphi$ $\left(\varphi^{+} / \varphi^{-}\right)$. Data sets [W27] (4224 observations) and [M27] (972 observations), both for VWI and EWI. Estimated standard errors are in parentheses.

\begin{tabular}{|c|c|c|c|c|c|c|c|c|}
\hline \multicolumn{9}{|c|}{ VWI: 1927-2007 (weekly) } \\
\hline Model & $\beta$ & $\eta$ & $\varphi\left(\varphi^{+} / \varphi^{-}\right)$ & $h_{\mathrm{WEEK} 1}$ & $\lambda_{1} \cdot 10^{3}$ & $\lambda_{2}$ & AIC & BIC \\
\hline asyV & $0.022(0.003)$ & $5.329(0.501)$ & $2.650(0.377) / 6.437(0.420)$ & $0.708(0.122)$ & $1.634(0.419)$ & $0.345(0.643)$ & $-21,098.9$ & $-21,054.4$ \\
\hline asyU & $0.023(0.003)$ & $5.521(0.503)$ & $2.757(0.381) / 6.669(0.434)$ & & $1.672(0.427)$ & $0.329(0.635)$ & $-21,097.4$ & $-21,059.3$ \\
\hline V & $0.019(0.003)$ & $5.050(0.502)$ & $4.461(0.491)$ & $0.699(0.120)$ & $1.604(0.404)$ & $1.086(0.948)$ & $-21,061.7$ & $-21,023.6$ \\
\hline $\mathrm{U}$ & $0.020(0.003)$ & $5.214(0.505)$ & $4.610(0.517)$ & & $1.606(0.404)$ & $1.113(0.946)$ & $-21,059.9$ & $-21,028.1$ \\
\hline
\end{tabular}

VWI: 1927-2007 (monthly)

\begin{tabular}{|c|c|c|c|c|c|c|c|c|}
\hline Model & $\beta$ & $\eta$ & $\varphi\left(\varphi^{+} / \varphi^{-}\right)$ & $h_{\mathrm{JAN}}$ & $\lambda_{1} \cdot 10^{3}$ & $\lambda_{2}$ & AIC & BIC \\
\hline asyV & $0.012(0.003)$ & $1.831(0.223)$ & $1.282(0.194) / 1.822(0.206)$ & $0.571(0.100)$ & $5.183(2.097)$ & $1.066(0.864)$ & -3221.5 & -3187.3 \\
\hline asyU & $0.010(0.003)$ & $1.755(0.265)$ & $1.239(0.189) / 1.792(0.201)$ & & $5.715(2.115)$ & $0.895(0.851)$ & -3214.5 & -3185.2 \\
\hline V & $0.011(0.003)$ & $1.830(0.220)$ & $1.560(0.229)$ & $0.562(0.096)$ & $4.667(2.061)$ & $1.390(0.902)$ & -3223.8 & -3194.5 \\
\hline $\mathrm{U}$ & $0.010(0.003)$ & $1.749(0.268)$ & $1.506(0.257)$ & & $5.452(2.088)$ & $1.060(0.919)$ & -3216.2 & -3191.8 \\
\hline
\end{tabular}

EWI: 1927-2007 (weekly)

\begin{tabular}{|c|c|c|c|c|c|c|c|c|}
\hline Model & $\beta$ & $\eta$ & $\varphi\left(\varphi^{+} / \varphi^{-}\right)$ & $h_{\text {WEEK1 }}$ & $\lambda_{1} \cdot 10^{3}$ & $\lambda_{2}$ & AIC & BIC \\
\hline asyV & $0.032(0.004)$ & $8.258(0.542)$ & $6.568(0.389) / 10.10(0.437)$ & $1.157(0.272)$ & $2.593(0.402)$ & $2.201(0.578)$ & $-20,596.9$ & $-20,552.4$ \\
\hline asyU & $0.032(0.004)$ & $8.311(0.538)$ & $6.602(0.391) / 10.20(0.442)$ & & $2.735(0.408)$ & $1.979(0.562)$ & $-20,598.4$ & $-20,560.3$ \\
\hline V & $0.032(0.004)$ & $7.565(0.460)$ & $7.081(0.456)$ & $1.088(0.239)$ & $2.439(0.388)$ & $3.263(0.737)$ & $-20,572.2$ & $-20,534.1$ \\
\hline $\mathrm{U}$ & $0.031(0.004)$ & $7.501(0.474)$ & $7.017(0.447)$ & & $2.443(0.389)$ & $3.250(0.738)$ & $-20,574.0$ & $-20,542.3$ \\
\hline
\end{tabular}

EWI: 1927-2007 (monthly)

\begin{tabular}{|c|c|c|c|c|c|c|c|c|}
\hline Model & $\beta$ & $\eta$ & $\varphi\left(\varphi^{+} / \varphi^{-}\right)$ & $h_{\mathrm{JAN}}$ & $\lambda_{1} \cdot 10^{3}$ & $\lambda_{2}$ & AIC & BIC \\
\hline asyV & $0.019(0.005)$ & $1.884(0.316)$ & $1.248(0.242) / 1.940(0.263)$ & $0.635(0.130)$ & $6.981(2.721)$ & $2.477(0.684)$ & -2763.3 & -2729.1 \\
\hline asyU & $0.019(0.005)$ & $1.883(0.319)$ & $1.220(0.238) / 1.978(0.274)$ & & $6.061(2.687)$ & $2.646(0.673)$ & -2759.4 & -2730.2 \\
\hline V & $0.022(0.005)$ & $2.011(0.283)$ & $1.671(0.270)$ & $0.583(0.119)$ & $6.730(2.691)$ & $2.660(0.721)$ & -2761.7 & -2732.4 \\
\hline $\mathrm{U}$ & $0.020(0.006)$ & $1.919(0.341)$ & $1.592(0.310)$ & & $5.866(2.652)$ & $2.787(0.706)$ & -2756.6 & -2732.2 \\
\hline
\end{tabular}

GARCH competitors (GARCH, QGARCH and GJRGARCH): in no case do we find a significant value of $\hat{\lambda}_{2}$ and the results scale up to reasonable values of the equity risk premium.

\section{Conclusion}

The relationship of the market risk-premium to risk is central to our understanding of Finance (Merton, 1980). Stochastic volatility models are a natural choice with which to model risk, yet, when this has been done, results have been disappointing. In a wide variety of studies, the literature has found, in varying degrees of certitude, evidence of positive, negative and no relationship of return to risk. Lundblad (2007) has argued that the disappointing results are the result of the low statistical power of small samples. Seeking to overcome the difficulties of sample size, he uses approximately two centuries of monthly data and finds evidence supporting a positive relationship between the market risk premium and risk.

In keeping with these endeavours, this paper has also sought to analyse Merton's proposition that returns are related to volatility, using a stochastic volatility model. We have concentrated on the application of the continuous time COGARCH model of Klüppelberg et al. (2004). While we are aware that it is just one of many competing models of stochastic volatility, we argue that it is highly appropriate to our analysis, which has focussed on daily data. COGARCH, as we have emphasised, provides an appropriate methodology as it facilitates rigorous examination of features of the data such as daily seasonalities and the effect of discontinuities (such as weekends). Our use of daily data goes some way to addressing Lundblad's (2007) concern that a large dataset is required to validly assess Merton's proposition.

Our analysis has, on balance, not provided support for Merton's proposition. In order to align with previous studies using discrete time GARCH models, we extended the COGARCH formulation to consider asymmetric responses of returns to risk, and these extensions proved to give the optimal model, among the COGARCH class, for our data. The asymmetric COGARCH models failed to find support for Merton's proposed positive relationship of return to risk (both for the value-weighted and equal-weighted indices we study). On the other hand, the nested COGARCH model not taking asymmetry into account provides significant support for Merton's proposition, as does a model ignoring weekend effects in the data. These results emphasise the importance of considering asymmetry and non-equal spacing in data when modelling the risk-return relationship using stochastic volatility methodologies. They also remind us of the sensitivity of the examination of the risk-return tradeoff to the specification of the models used to examine the relationship.

We draw two major conclusions from our analysis. Firstly, we have demonstrated the flexibility of COGARCH in dealing with unequally spaced data; in particular, we have demonstrated how COGARCH may be adapted to account for asymmetry. Due to its 
flexibility, COGARCH is a very useful tool for financial econometricians. Secondly, and perhaps most importantly, we have added substantially to the discussion of Merton's proposition that there should be a positive relationship of return to risk. While we do indeed estimate a positive relationship in all our analyses, our most favored model does not provide statistically significant evidence of such. Consequently our paper has to be considered as adding to but not resolving the mixed, and often weak, evidence previously found for (and, sometimes, against) the proposition. Once again, we are thrown back on time-honored explanations: is the signal to noise ratio too weak to be observed, even in an extensive analysis such as ours? Does it in fact exist? It is sad to reflect that a belief in a positive risk-return trade-off still appears to be as much a matter of faith as a well-established and scientifically supported proposition.

\section{Acknowledgements}

We are extremely grateful for the careful reading and insightful comments and suggestions provided by the referee of this paper; these thoughts have helped us improve the paper significantly. We are also grateful for comments made by the editor Christian C.P. Wolff; they have assisted in the polishing of this paper. The research was partially supported by ARC grant DP1092502.

\section{Appendix A. Estimation via the PML method}

Both the pure COGARCH model and extensions (such as our inclusion of extra regression variables) can be estimated by the pseudo-maximum-likelihood (PML) method set out in Maller et al. (2008). This produces estimates for the three COGARCH parameters, the regression parameters and any parameters measuring effects of exogenous variables, where included, as well as standard errors for all parameters. Since the extension of the PML estimation method for the COGARCH to our regression analysis is straightforward, we describe this method only for the pure COGARCH model. The following description is extracted from Maller et al. (2008), which see for further details.

The method is based on an approximation of the COGARCH model by a sequence of discrete-time GARCH models. To describe the convergence of the discrete to the continuous time GARCH, we have to introduce an extra index, $n$. Thus, starting with a finite interval [0,T], T>0, take deterministic sequences $\left(N_{n}\right)_{n \geq 1}$ with $\lim _{n \rightarrow \infty} N_{n}=\infty$ and $0=t_{0}(n)<t_{1}(n)<\cdots<t_{N_{n}}(n)=T$, and, for each $n=1,2, \ldots$, divide $[0, T]$ into $N_{n}$ subintervals of length $\Delta t_{i}(n):=t_{i}(n)-t_{i-1}(n)$, for $i=1,2, \ldots, N_{n}$. Define, for each $n=1,2, \ldots$, a discrete time process $\left(G_{i, n}\right)_{i=1, \ldots, N_{n}}$ satisfying

$$
G_{i, n}=G_{i-1, n}+\rho_{i-1, n} \sqrt{\Delta t_{i}(n)} \varepsilon_{i, n}, i=1,2, \ldots, N_{n}
$$

where $G_{0, n}=G(0)=0$, the variance $\rho_{i, n}^{2}$ follows the recursion

$$
\rho_{i, n}^{2}=\beta \Delta t_{i}(n)+\left(1+\varphi \Delta t_{i}(n) \varepsilon_{i, n}^{2}\right) e^{-\eta \Delta t_{i}(n)} \rho_{i-1, n}^{2}, i=1,2, \ldots, N_{n},
$$

and the innovations $\left(\varepsilon_{i, n}\right)_{i=1, \ldots, N_{n}}, n=1,2, \ldots$, are constructed from the Lévy process $L$ using a "first jump" approximation developed by Szimayer and Maller (2007). The discrete time processes $G_{\cdot, n}$ and $\rho_{\cdot, n}^{2}$ are embedded into continuous time versions $G_{n}$ and $\rho_{n}^{2}$ defined by

$$
G_{n}(t):=G_{i, n} \text { and } \rho_{n}^{2}(t):=\rho_{i, n}^{2} \text {, when } t \in\left[t_{i-1}(n), t_{i}(n)\right), 0 \leq t \leq T \text {, }
$$

with $G_{n}(0)=0$.

Assuming $\max _{i=1, \ldots, N_{n}} \Delta t_{i}(n) \rightarrow 0$ as $n \rightarrow \infty$, a main result of Maller et al. (2008) is that the discretised, piecewise constant processes $\left(G_{n}, \rho_{n}^{2}\right)_{n \geq 1}$ defined by Eq. (12) converge as $n \rightarrow \infty$ in distribution in the Skorohod topology on $D[0, T]$ (the space of càdlàg real-valued stochastic processes on $[0, T]$ ) to the continuous time processes $\left(G, \rho^{2}\right)$ defined by Eqs. (2) and (3). Practically, this means that for a very large data set such as we have, the fitted discrete time process will very closely approximate the underlying continuous time COGARCH model, with a corresponding close approximation of the parameters.

Because $\left(\rho^{2}(t)\right)_{t \geq 0}$ is Markovian, the return $Y_{i}$ defined in Eq. (4) is conditionally independent of $Y_{i-1}, Y_{i-2}, \ldots$, given the natural filtration of the Lévy process $L$, To apply the PML method, we assume at first that the $Y_{i}$ are conditionally distributed as $N\left(0, \sigma_{i}^{2}\right)$, then use recursive conditioning involving a GARCH-type recursion for the variance process to write a pseudo-log-likelihood function for $Y_{1}, Y_{2}, \ldots, Y_{N}$ as

$$
\mathcal{L}_{N}=\mathcal{L}_{N}(\beta, \varphi, \eta)=-\frac{1}{2} \sum_{i=1}^{N}\left(\frac{Y_{i}^{2}}{\sigma_{i}^{2}}\right)-\frac{1}{2} \sum_{i=1}^{N} \log \left(\sigma_{i}^{2}\right)-\frac{N}{2} \log (2 \pi)
$$


c.f.Eq. (3.3) of Maller et al. (2008). We must substitute in Eq. (13) a calculable quantity for $\sigma_{i}^{2}$, hence we need such for $\rho^{2}\left(t_{i-1}\right)$ in Eq. (5). For this, we discretise the continuous time volatility process just as was done in Maller et al. (2008). Thus we let

$$
\rho_{i}^{2}=\beta \Delta t_{i}+e^{-\eta \Delta t_{i}} \rho_{i-1}^{2}+\varphi e^{-\eta \Delta t_{i}} Y_{i}^{2}
$$

Eq. (14) is a GARCH-type recursion, so, after substituting $\rho_{i-1}^{2}$ for $\rho^{2}\left(t_{i-1}\right)$ in Eq. (5), and the resulting modified $\sigma_{i}^{2}$ in Eq. (13), we can think of Eq. (13) as the pseudo-log-likelihood function for fitting a GARCH model to the unequally spaced series. Taking the stationary value $\beta /(\eta-\varphi)$ as starting value for $\rho^{2}(0)$, we can maximise $L_{N}$ to get PMLEs of $(\beta, \eta, \varphi)$ and estimates of their standard deviations.

Eq. (14) specifies the recursion when returns are treated symmetrically in the sense that positive and negative returns feedback equally into volatility. To specify the asymmetric COGARCH, we modify the recursion as follows. Recall that $Y_{i}=R_{i}-r_{f, i}$, and define $Z_{i}:=Y_{i}-\left(\lambda_{1}+\lambda_{2} \sigma_{i}^{2}\right)$. Then proceed as follows: if $Z_{i} \geq 0$, use the recursion equations

$$
\rho_{i}^{2}=\beta \Delta t_{i}+e^{-\eta \Delta t_{i}} \rho_{i-1}^{2}+\varphi^{+} e^{-\eta \Delta t_{i}} Z_{i}^{2}
$$

and

$$
\sigma_{i+1}^{2}=\left(\rho_{i}^{2}-\frac{\beta}{\eta-\varphi^{+}}\right)\left(\frac{e^{\left(\eta-\varphi^{+}\right) \Delta t_{i+1}}-1}{\eta-\varphi^{+}}\right)+\frac{\beta \Delta t_{i+1}}{\eta-\varphi^{+}}
$$

if $Z_{i}<0$, use the recursion equations

$$
\rho_{i}^{2}=\beta \Delta t_{i}+e^{-\eta \Delta t_{i}} \rho_{i-1}^{2}+\varphi^{-} e^{-\eta \Delta t_{i}} Z_{i}^{2}
$$

and

$$
\sigma_{i+1}^{2}=\left(\rho_{i}^{2}-\frac{\beta}{\eta-\varphi^{-}}\right)\left(\frac{e^{\left(\eta-\varphi^{-}\right) \Delta t_{i+1}}-1}{\eta-\varphi^{-}}\right)+\frac{\beta \Delta t_{i+1}}{\eta-\varphi^{-}}
$$

For those models using the virtual time scale, replace $\Delta t_{i}$ by $\Delta \tau_{i}$. Note the similarity to the GJR-GARCH model which we considered in Section 4.2. The term $\varphi^{+} e^{-\eta \Delta t_{i}}$ corresponds, approximately, to $c_{\mathrm{GJR}}$, and $\varphi^{-} e^{-\eta \Delta t_{i}}$ corresponds to $c_{\mathrm{GJR}}+e_{\mathrm{GJR}}$.

\section{References}

Andersen, T., Bollerslev, T., 1998. Answering the skeptics: yes, standard volatility models do provide accurate forecasts. Int. Econ. Rev. $39,885-905$. Ang, A., Liu, J., 2007. Risk, return, and dividends. J. Financ. Econ. 85, 1-38.

Ang, A., Hodrick, R.J., Xing, Y., Zhang, X., 2006. The cross-section of volatility and expected returns. J. Finance 61, 259-299.

Applebaum, D., 2004. Lévy Processes and Stochastic Calculus. Cambridge Studies in Advanced Mathematics 93. Cambridge University Press, Cambridge.

Baillie, R.T., DeGennaro, R., 1990. Stock returns and volatility. J. Financ. Quant. Anal. 25, 203-214.

Banz, R.W., 1981. The relationship between return and market value of common stocks. J. Financ. Econ. 9, 3-18.

Bekaert, G., Wu, G., 2000. Asymmetric volatility and risk in equity markets. Rev. Financ. Stud. 13, 1-42.

Berk, J.B., 1995. A critique of size related anomalies. Rev. Financ. Stud. 9, 275-286.

Bertoin, J., 1996. Lévy Processes. Cambridge University Press, Cambridge.

Bhardwaj, R.K., Brooks, L.D., 1992. The January Anomaly: effect of low share price, transaction costs, and bid-ask bias. J. Finance 47, $553-575$.

Brockman, P., Michayluk, D., 1997. The Holiday Anomaly: an investigation of firm size versus share price effects. Q. J. Bus. Econ. $36,23-35$.

Brockwell, P., Chadraa, E., Lindner, A., 2006. Continuous-time GARCH processes. Ann. Appl. Probab. 16, 790-826.

Brown, P., Keim, D., Kleidon, A., Marsh, T., 1983. Stock return seasonalities and the tax-loss selling hypothesis: analysis of the arguments and Australian evidence. J. Financ. Econ. 12, 105-127.

Campbell, J.Y., Hentschel, L., 1992. An asymmetric model of changing volatility in stock returns. J. Financ. Econ. 31, 281-318.

Chen, N.F., 1991. Financial Investment Opportunities and the Macroeconomy. Journal of Finance 46, 529-554.

Cox, J.C., Ingersoll Jr., J.E., Ross, S.A., 1985. A theory of the term structure of interest rates. Econometrica 53, $385-408$.

Durand, R.B., Lim, D., Zumwalt, J.K., 2011. Fear and the Fama-French Factors. Working paper available at SSRN: http://ssrn.com/abstract = 965587.

Erickson, J., Wang, K., Li, Y., 1997. A New Look at the Monday Effect. Journal of Finance 52, 2171-2186.

Fama, E.F., French, K.R., 1993. Common risk factors in the returns on stocks and bonds. J. Financ. Econ. 33, 3-57.

Fasen, V., Klüppelberg, C., Lindner, A., 2005. Extremal behavior of stochastic volatility models. In: Shiryaev, A.N., Grossinho, M.D.R., Oliviera, P.E., Esquivel, M.L. (Eds.), Stochastic Finance. Springer, New York.

Flannery, M., Protopapadakis, A.A., 2002. Macroeconomic factors do influence aggregate stock returns. Rev. Financ. Stud. 15, $751-782$.

French, K.R., 1980. Stock returns and the weekend effect. J. Financ. Econ. 8, 55-69.

French, K., Schwert, G.W., Stambaugh, R., 1987. Expected stock returns and volatility. J. Financ. Econ. 19, 3-30.

Ghysels, E., Santa-Clara, P., Valkanov, R., 2005. There is a risk-return trade-off after all. J. Financ. Econ. 76, 509-548.

Gibbons, M., Hess, P., 1981. Day of the week effects and asset returns. J. Bus. 54, 579-596.

Glosten, L.R., Jagannathan, R., Runkle, D.R., 1993. On the relation between the expected value and the volatility of the nominal excess returns on stocks. J. Finance 48, 1779-1801.

Guo, H., Whitelaw, R., 2006. Uncovering the risk-return relation in the stock market. J. Finance 61, 1433-1463. 
James, C., Edmister, R.O., 1983. The relation between common stock returns trading activity and market value. J. Finance 38, $1075-1086$.

Jorion, P., Goetzmann, W.N., 1999. Global stock markets in the twentieth century. J. Finance 54, 953-980.

Keim, D., 1983. Size-related anomalies and stock return seasonality: further empirical evidence. J. Financ. Econ. $25,75-97$.

Klüppelberg, C., Lindner, A., Maller, R.A., 2004. A continuous time GARCH process driven by a Lévy process: stationarity and second order behaviour. J. Appl. Probab. 41, 601-622.

Klüppelberg, C., Lindner, A., Maller, R.A., 2006. Continuous time volatility modelling: COGARCH versus Ornstein-Uhlenbeck models. In: Kabanov, Y., Lipster, R., Stoyanov, J. (Eds.), From Stochastic Calculus to Mathematical Finance. : The Shiryaev Festschrift. Springer, Berlin.

Lewellen, J., Nagel, S., 2006. The conditional CAPM does not explain asset-pricing anomalies. J. Financ. Econ. 82, 289-314.

Lundblad, Ch., 2007. The risk-return trade-off in the long run: 1836-2003. J. Financ. Econ. 85, 123-150.

Maller, R.A., Müller, G., Szimayer, A., 2008. GARCH modelling in continuous time for irregularly spaced time series data. Bernoulli 14, 519-542.

Merton, R., 1973. An intertemporal capital asset pricing model. Econometrica 41, 867-887.

Merton, R., 1980. On estimating the expected return on the market: an exploratory investigation. J. Financ. Econ. 8, $323-361$.

Maller, R.A., Müller, G., 2010. On the Residuals of GARCH(1,1) and Extensions when Estimated by Maximum Likelihood. Technical Report. Australian National University and Technische Universität München.

Müller, G., Durand, R.B., Maller, R.A., Klüppelberg, C., 2009. Analysis of stock market volatility by continuous-time GARCH models. In: Gregoriou, G.N. (Ed.), Stock Market Volatility. London, Chapman and Hall-CRC/Taylor and Francis.

Nelson, D.B., 1991. Conditional heteroskedasticity in asset returns: a new approach. Econometrica 59, 347-370.

Pástor, L., Sinha, M., Swaminathan, B., 2008. Estimating the intertemporal risk-return tradeoff using the implied cost of capital. J. Finance 63, $2859-2897$.

Platen, E., Sidorowicz, R., 2007. Empirical evidence on Student-t log-returns of diversified world indices. J. Statist. Theory Pract. 2, $233-251$.

Protter, P., 2005. Stochastic Integration and Differential Equations, 2nd ed. Springer, Heidelberg.

Reinganum, M., Shapiro, A., 1987. The anomalous stock market behavior of small firms in January: empirical tests for the tax-loss selling effects. Journal of Business 60, 281-295.

Sato, K., 1999. Lévy Processes and Infinitely Divisible Distributions. Cambridge University Press, Cambridge.

Scruggs, J.T., 1998. Resolving the puzzling intertemporal relation between the market risk premium and conditional market variance: a two-factor approach. J. Finance 53, 575-603.

Stelzer, R., 2009. Multivariate GARCH $(1,1)$ Processes. Bernoulli, to appear.

Szimayer, A., Maller, R.A., 2007. Finite approximation schemes for Lévy processes, and their application to optimal stopping problems. Stoch. Proc. Appl. 117, $1422-1447$.

Welch, I., 2000. Views of financial economists on the equity premium and on professional controversies. J. Bus. 73, $501-537$. 